OPEN ACCESS

Edited by:

James Moon,

University of Michigan, United States

Reviewed by:

Peter M. Van Endert,

Institut National de la Santé et de la

Recherche Médicale (INSERM),

France

Bergithe Eikeland Oftedal,

University of Bergen, Norway

*Correspondence:

Pere Santamaria

psantama@ucalgary.ca

Specialty section:

This article was submitted to Immunological Tolerance and Regulation,

a section of the journal

Frontiers in Immunology

Received: 27 October 2020 Accepted: 09 December 2020

Published: 26 January 2021

Citation:

Serra $P$ and Santamaria $P$ (2021)

Peptide-MHC-Based

Nanomedicines for the Treatment

of Autoimmunity: Engineering,

Mechanisms, and Diseases.

Front. Immunol. 11:621774.

doi: 10.3389/fimmu.2020.621774

\section{Peptide-MHC-Based Nanomedicines for the Treatment of Autoimmunity: Engineering, Mechanisms, and Diseases}

\author{
Pau Serra ${ }^{1}$ and Pere Santamaria ${ }^{1,2 *}$ \\ 1 Institut D'Investigacions Biomèdiques August Pi i Sunyer, Barcelona, Spain, 2 Julia McFarlane Diabetes Research Centre \\ (JMDRC) and Department of Microbiology, Immunology and Infectious Diseases, Snyder Institute for Chronic Diseases and \\ Hotchkiss Brain Institute, Cumming School of Medicine, University of Calgary, Calgary, AB, Canada
}

The development of autoimmunity results from a breakdown of immunoregulation and involves cellularly complex immune responses against broad repertoires of epitope specificities. As a result, selective targeting of specific effector autoreactive T- or B-cells is not a realistic therapeutic option for most autoimmune diseases. Induction of autoantigen-specific regulatory T-cells capable of effecting bystander (dominant), yet tissue-specific, immunoregulation has thus emerged as a preferred therapeutic alternative. We have shown that peptide-major histocompatibility complex (pMHC)based nanomedicines can re-program cognate autoantigen-experienced T-cells into disease-suppressing regulatory T-cells, which in turn elicit the formation of complex regulatory cell networks capable of comprehensively suppressing organ-specific autoimmunity without impairing normal immunity. Here, we summarize the various pMHC-based nanomedicines and disease models tested to date, the engineering principles underpinning the pharmacodynamic and therapeutic potency of these compounds, and the underlying mechanisms of action.

Keywords: peptide-major histocompatibility complex molecules, nanoparticles, autoimmune diseases, T-cell re-programming, T-regulatory type 1 cells

\section{INTRODUCTION}

The development of autoimmune disease results from dysregulated immune responses to self that are triggered by ill-defined environmental cues in genetically predisposed individuals. Such immune responses lead to the activation and recruitment of effector autoreactive T-cells into specific tissues/ organs, the recruitment of additional inflammatory cell types to the site, chronic inflammation and, eventually, tissue/organ dysfunction and/or destruction. Given the autoantigenic complexity of most autoimmune disorders, targeting of effector autoreactive T-cell specificities is not a realistic therapeutic option for the treatment of these diseases. An alternative includes promoting the formation and/or expansion of regulatory autoreactive T-cell clonotypes capable of effecting bystander immunoregulation (against the many non-cognate autoantigenic epitopes that are targeted in the course of a specific disease). Several approaches that are potentially capable of 
eliciting bystander immunoregulation have been described over the last decade, but the mechanisms of action of some of these remain unclear and their therapeutic efficacy has not been thoroughly tested in non-contrived models of spontaneous, polyclonal autoimmunity [reviewed in (1)].

We have shown that profound and sustained ligation of antigen receptors on cognate effector autoreactive $\mathrm{T}$-cells by nanoparticles (NPs) displaying multiple copies of diseaserelevant peptide-MHC class I or class II complexes (pMHC$\mathrm{NP}$ ) can trigger their differentiation into regulatory T-cells in vivo. Upon pMHC-NP-induced expansion, these cognate, monospecific autoreactive $\mathrm{T}$-cells elicit self-sustaining regulatory cell networks that efficiently suppress polyclonal autoreactive T-cell responses in several murine models of autoimmunity, without compromising normal immunity. In this mini-review, we discuss the key engineering principles behind the pharmacodynamic activity of these compounds, the mechanisms underlying their therapeutic activity, and the disease models in which we have documented efficacy (Table 1).

\section{pMHCI-NPS AS TRIGGERS OF AUTOREGULATORY MEMORY-LIKE CD8+ T-CELL EXPANSION}

Our initial attempts at developing an antigen-specific therapeutic approach for type 1 diabetes (T1D) aimed at triggering the deletion of a highly prevalent and diabetogenic CD8+ T-cell population specific for residues 206-214 of the islet-specific glucose-6-phosphatase catalytic subunit-related protein $\left(\right.$ IGRP $\left._{206-214}\right)$. This T-cell specificity plays a significant role in the progression of islet inflammation to beta cell destruction in NOD mice (6). Certain IGRP $_{206-214}$ mimotopes could blunt disease progression in pre-diabetic mice by selectively triggering the deletion of high-avidity IGRP $_{206-214}$-reactive clonotypes, while sparing their low-avidity counterparts (6). Surprisingly, treatment of pre-diabetic mice with the natural ligand or with super-agonistic mimotopes, which simultaneously deleted both high- and low-avidity clonotypes, was devoid of therapeutic activity (7). Subsequent experiments in T-cell receptor (TCR)-transgenic NOD mice expressing either low or high-affinity TCRs for IGRP $206-214$ demonstrated that the antidiabetogenic effect of protective mimotopes was mediated by the low-avidity T-cell pool, which accumulated in the islets of Langerhans and presumably shielded beta cells from beta cell destruction by other autoreactive T-cell specificities $(7,8)$.

These observations exposed important limitations of mimotope-based immunotherapies. Namely, that complete deletion of individual mono-specific T-cell specificities is insufficient to blunt the progression of antigenically complex autoimmune disorders, and that the therapeutic success of antigen/peptide therapy hinges on the identification of optimal amino acid sequences, doses, and therapeutic regimens capable of eliciting the type of bystander regulation described above (9). Unfortunately, accurate prediction of the pharmacodynamic and therapeutic effects of specific peptide ligands in vivo is not currently possible or straightforward, thus hindering the translation of this approach for the treatment of human autoimmune disorders.

In an attempt to overcome these challenges, we sought to blunt the progression of T1D in NOD mice by simultaneously deleting multiple epitope T-cell specificities at once. We reasoned that, by virtue of their higher avidity for cognate T-cells and lack of co-stimulatory potential, NPs coated with multiple copies of disease-relevant pMHC class I (pMHCI) complexes, should be able to efficiently deplete cognate CD8+ clonotypes over a broad dose range. We further reasoned that, if this hypothesis were true, combinations of pMHCI-NPs targeting different CD8+ Tcell specificities should be able to substantially reduce the pool of beta cell killing effectors. Surprisingly, although treatment of NOD mice with the multi-specific pool of pMHCI-NPs had therapeutic effects, so did NPs exclusively displaying the $\mathrm{IGRP}_{206-214} / \mathrm{K}^{\mathrm{d}}$ pMHCI (Table 1). Detailed examination of the therapeutic effects and mechanistic underpinnings of monospecific pMHCI-NP therapy revealed that the therapeutic effect of these compounds was mediated by expansion of cognate lowavidity memory-like CD8+ T-cells with dominant regulatory potential. These memory-like autoregulatory CD8+ T-cells suppressed the activation of non-cognate autoreactive $\mathrm{T}$-cell specificities by both suppressing and killing autoantigen-loaded professional antigen-presenting cells (APCs) in the pancreatic islets and pancreas-draining lymph nodes in an antigen-specific manner (2).

\section{THERAPEUTIC PROPERTIES OF pMHCII- NPS DISPLAYING TISSUE-SPECIFIC EPITOPES}

The allelic complexity MHC class I loci in humans limits the translational significance of pMHCI-NPs for human immunotherapy, as numerous compounds would need to be developed to treat a significant fraction of the patient population for any given autoimmune disease.

Our work with pMHCI-NPs suggested that treatment with these compounds harnesses a naturally-occurring negative feedback regulatory loop that might have arisen during natural evolution to oppose the progression of autoimmune inflammation. In turn, this idea suggested that such negative feedback regulatory loops might also exist in the autoreactive CD4+ T-cell compartment. This hypothesis predicted that treatment of autoimmune diseaseaffected mice with pMHCII-NPs would elicit the formation and/ or expansion of autoantigen-specific regulatory CD4+ T-cells. Since there are strong associations between human autoimmune diseases and certain HLA class II types, and CD4+ T-cells play a central role in the initiation, progression and maintenance of most, if not all autoimmune diseases, we reasoned that these pMHCII-based compounds would have superior translational significance than their pMHCI-based counterparts.

We demonstrated that various murine T1D-relevant pMHCII-NPs (displaying BDC2.5mi/IA ${ }^{\mathrm{g} 7}, \mathrm{IGRP}_{128-145} / \mathrm{IA}^{\mathrm{g} 7}$ or $\mathrm{IGRP}_{4-22} / \mathrm{IA}^{\mathrm{g} 7}$ ) could stably restore normoglycemia in 
TABLE 1 | pMHC-based nanomedicines and models.

\begin{tabular}{|c|c|c|c|c|c|c|}
\hline pMHC-nanomedicines & Disease target & Animal model & Disease tested & PD activity & Ther. activity & Reference \\
\hline \multirow{2}{*}{ NRP-V7-K $-\mathrm{NP}$} & & $\mathrm{B} 10 \cdot \mathrm{H} 2^{97}$ & None & - & - & (2) \\
\hline & & NOD.G6pc2 $2^{\text {K209A-F213A }}$ & T1D & - & - & (2) \\
\hline IGRP $_{206-214}-K^{d}-N P$ & $\mathrm{TID}$ & NOD & TID & + & + & (2) \\
\hline MimA2(DMK $\left.{ }_{138-146}\right)-D^{b}-N P$ & TID & NOD & TID & + & + & (2) \\
\hline TUM-K ${ }^{d}-N P$ & None & NOD & TID & - & - & (2) \\
\hline hIGRP ${ }_{265-273}-\mathrm{A} 2 \mathrm{~K}^{\mathrm{b}}-\mathrm{NP}$ & TID & NOD.HHD & TID & + & + & (2) \\
\hline \multirow[t]{3}{*}{$\mathrm{BDC} 2.5 \mathrm{mi} / \mathrm{IA}^{\mathrm{g}^{7}}-\mathrm{NP}$} & TID & NOD & $\mathrm{TID}$ & + & + & (3) \\
\hline & & NOD G6pc2 $2^{-/-}$ & TID & + & + & (3) \\
\hline & & NOD.c3c4 & PBC & - & - & (4) \\
\hline \multirow[t]{2}{*}{$\mathrm{IGRP}_{4-22} / \mathrm{IA}^{97}-\mathrm{NP}$} & TID & NOD & TID & + & + & (3) \\
\hline & & NOD G6pc2 $2^{-/-}$ & TID & - & - & (3) \\
\hline IGRP $_{128-145} / \mathrm{IA}^{\mathrm{g} 7}-\mathrm{NP}$ & $\mathrm{TID}$ & NOD & TID & + & + & (3) \\
\hline $\mathrm{pMOG}_{38-49} / \mathrm{A}^{\mathrm{b}}-\mathrm{NP}$ & EAE & EAE in C57BL/6 & EAE (pMOG $\left.{ }_{35-55}\right)$ & + & + & (3) \\
\hline hPLP $175-192 / D R 4-I E-N P$ & EAE & C57BL/6 IAb ${ }^{\text {null }}$ HLA-DR4-IE & EAE (hPLP $175-192)$ & + & + & (3) \\
\hline \multirow[t]{3}{*}{ hMOGg7-108/DR4-IE-NP } & EAE & C57BL/6 IAb $b^{\text {null }} H L A-D R 4-I E$ & EAE $\left(h M O G_{97-108}\right)$ & + & + & (3) \\
\hline & & C57BL/6 IAb ${ }^{\text {null }}$ HLA-DR4-IE & EAE (hPLP $175-192)$ & + & + & (3) \\
\hline & & C57BL/10.M HLA-DR4-IE & $\mathrm{ClA}$ & - & - & (3) \\
\hline \multirow[t]{2}{*}{$\mathrm{mCll}_{259-273} / \mathrm{DR} 4-\mathrm{IE}-\mathrm{NP}$} & $\mathrm{ClA}$ & C57BL/10.M HLA-DR4-IE & $\mathrm{ClA}$ & + & + & (3) \\
\hline & $\mathrm{ClA}$ & C57BL/6 IA $b^{\text {null }} H L A-D R 4-I E$ & EAE (hPLP $175-192)$ & - & - & (3) \\
\hline $\mathrm{MOG}_{36-50} / \mathrm{IA}^{97}-\mathrm{NPS}$ & EAE & EAE in NOD & EAE $\left(M^{\prime} G_{35-55}\right)$ & + & + & (4) \\
\hline \multirow[t]{5}{*}{$\mathrm{PDC}_{166-181^{-}-\mathrm{A}^{97}-\mathrm{NPS}}$} & PBC & NOD.c3c4 & $\mathrm{PBC}$ & + & + & (4) \\
\hline & & (NODxB6.lfng-ARE-Del-1-) F1 & PBC & + & + & (4) \\
\hline & & Ad-FTCD-AlH in NOD & $\mathrm{AlH}$ & + & + & (4) \\
\hline & & Abcb4-KO $\left(\mathrm{MDR3}^{-/-}\right)$ & PSC & + & + & (4) \\
\hline & & EAE in NOD & $\operatorname{EAE}\left(\mathrm{MOG}_{35-55}\right)$ & + & + & (4) \\
\hline \multirow{4}{*}{$\mathrm{BDC} 2.5 \mathrm{mi} / \mathrm{/A} \mathrm{A}^{\mathrm{9}}-\mathrm{NP}$} & & NOD.RIP-hDTR + DT & T1D & + & $\mathrm{N} / \mathrm{A}$ & (5) \\
\hline & & EAE in NOD & $\operatorname{EAE}\left(\mathrm{MOG}_{35-55}\right)$ & + & - & (5) \\
\hline & & Ad-FTCD-AlH in NOD & $\mathrm{AlH}$ & + & - & (5) \\
\hline & & $\mathrm{EAE}+\mathrm{PBC}$ in NOD.c3c4 & $\mathrm{EAE}\left(\mathrm{MOG}_{35-55}\right)$ and PBC & - & $\begin{array}{l}-(P B C) \\
-(E A E)\end{array}$ & (5) \\
\hline \multirow[t]{4}{*}{$\mathrm{PDC}_{166-181}-\mid \mathrm{A}^{\mathrm{g}}-\mathrm{NPS}$} & PBC & NOD & T1D & - & $\mathrm{N} / \mathrm{A}$ & (5) \\
\hline & & NOD.RIP-hDTR + DT & $\mathrm{T} 1 \mathrm{D}$ & + & $\mathrm{N} / \mathrm{A}$ & (5) \\
\hline & & EAE in NOD & $\operatorname{EAE}\left(\mathrm{MOG}_{35-55}\right)$ & + & + & (5) \\
\hline & & $\mathrm{EAE}+\mathrm{PBC}$ in NOD.c3c4 & $\mathrm{EAE}\left(\mathrm{MOG}_{35-55}\right)$ and PBC & + & $\begin{array}{l}+(\mathrm{PBC}) \\
-(\mathrm{EAE})\end{array}$ & (5) \\
\hline \multirow{4}{*}{$\mathrm{CYPD}_{398-412} / \mathrm{IA}^{\mathrm{g} 7}-\mathrm{NPs}$} & $\mathrm{AlH}$ & NOD & $\mathrm{T} 1 \mathrm{D}$ & - & $\mathrm{N} / \mathrm{A}$ & (5) \\
\hline & & NOD.RIP-hDTR + DT & T1D & + & $\mathrm{N} / \mathrm{A}$ & (5) \\
\hline & & EAE in NOD & $\operatorname{EAE}\left(\mathrm{MOG}_{35-55}\right)$ & + & + & (5) \\
\hline & & $\mathrm{EAE}+\mathrm{PBC}$ in NOD.c3c4 & $\mathrm{EAE}\left(\mathrm{MOG}_{35-55}\right)$ and PBC & + & $\begin{array}{l}+(\mathrm{PBC}) \\
-(\mathrm{EAE})\end{array}$ & (5) \\
\hline \multirow{3}{*}{$\mathrm{MOG}_{36-50} / \mathrm{IA}^{\mathrm{g}^{7}}$-NPs } & EAE & NOD.RIP-hDTR + DT & T1D & - & $\mathrm{N} / \mathrm{A}$ & (5) \\
\hline & & EAE in NOD & EAE $\left(M^{\prime} G_{35-55}\right)$ & + & + & (5) \\
\hline & & $\mathrm{EAE}+\mathrm{PBC}$ in NOD.c3c4 & $\operatorname{EAE}\left(\mathrm{MOG}_{35-55}\right)$ and PBC & + & $\begin{array}{l}-(\mathrm{PBC}) \\
-(\mathrm{EAE})\end{array}$ & (5) \\
\hline \multirow[t]{2}{*}{$\mathrm{pMOG}_{38-49} / \mathrm{A}^{\mathrm{b}}-\mathrm{NP}$} & EAE & EAE in C57BL/6 & EAE (pMOG $\left.{ }_{35-55}\right)$ & + & + & (5) \\
\hline & EAE & $\mathrm{EAE}+\mathrm{Ad}-\mathrm{CYPD}-\mathrm{AlH}$ in C57BL/6 & EAE $\left(\mathrm{pMOG}_{35-55}\right)$ and $\mathrm{AlH}$ & + & $\begin{array}{l}+(\mathrm{EAE}) \\
-(\mathrm{AlH})\end{array}$ & (5) \\
\hline
\end{tabular}


TABLE 1 | Continued

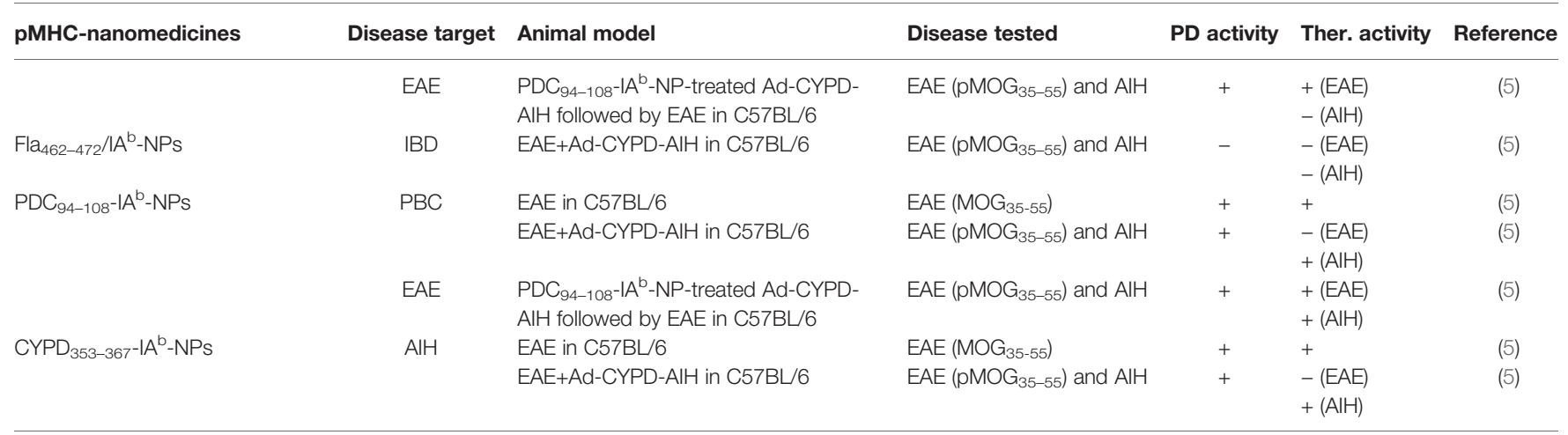

TID, Type 1 Diabetes; EAE, Experimental Autoimmune Encephalomyelitis; CIA, Collagen-Induced Arthritis; PBC, Primary Biliary Cholangitis; AlH, Autoimmune Hepatitis; PSC, Primary Sclerosing Cholangitis.

spontaneously diabetic NOD mice (Table 1). A similar outcome was obtained in wild-type C57BL/6 and HLA-DR4-transgenic C57BL/6 mice with experimental autoimmune encephalomyelitis (EAE, a murine model of multiple sclerosis). NPs displaying myelin oligodendrocyte glycoprotein $(\mathrm{MOG})_{38-49} / \mathrm{IA}^{\mathrm{b}}$ or human proteolipid protein (hPLP) ${ }_{175-192} / \mathrm{DR} 4$ complexes were able to reverse limb paralysis in these animals when administered at the peak of disease severity. Similar therapeutic effects were seen when using $\mathrm{hMOG}_{97-108} / \mathrm{DR}$-IE-NPs to treat $\mathrm{hPLP}_{175-192}$-induced EAE in HLA-DR4-transgenic C57BL/6 mice, demonstrating that the pMHCII displayed by these compounds need not have to target disease-initiating T-cells (Table 1). Likewise, NPs displaying mouse collagen II (mCII $)_{259-273} / \mathrm{DR} 4$ could reverse both joint swelling and destruction in HLA-DR4-transgenic C57BL/10.M mice immunized with bovine collagen (3) (Table 1). These therapeutic effects were disease-specific because $\mathrm{mCII}_{259-273} / \mathrm{DR} 4-\mathrm{NPs}$ and $\mathrm{hPLP}_{175-192} /$ DR4-NPs lacked therapeutic activity against EAE or collageninduced arthritis, respectively, in HLA-DR4-transgenic mice (3) (Table 1). Furthermore, they did not compromise the ability of the host to clear a systemic viral infection or to mount antibody responses against an experimental vaccine (3).

\section{PHARMACODYNAMIC ACTIVITY: PMHCII- NPS AS TRIGGERS OF T-REGULATORY TYPE 1 CELL FORMATION AND EXPANSION}

These therapeutic effects were invariably associated with systemic expansions of cognate CD4+ T-cells displaying a Tregulatory type 1 ( $\operatorname{Tr} 1)$-like phenotype and transcriptional profile, as compared to murine Tr1-like cells described elsewhere (10). Experiments in diabetic NOD mice lacking expression of the antigenic epitope displayed on the pMHCNP complex (hence lacking cognate epitope-experienced T-cells) demonstrated that the pharmacodynamic and therapeutic effects of both pMHCI- and pMHCII-NPs required the presence of autoantigen-experienced T-cells $(2,3)$ (Table 1). Clearly, these compounds target both naive and antigen-activated cognate
CD4+ T-cells, but do so with divergent consequences. Thus, whereas pMHC-NPs induce activation-induced cell death of naive T-cells, they trigger the expansion of memory-like low avidity autoregulatory CD8+ cells (pMHCI-NP) or the differentiation of autoantigen-experienced effectors into $\operatorname{Tr} 1$ cells instead (pMHCII-NP). The lack of co-stimulatory signals on pMHCII-NPs, the absolute need for co-stimulation in the survival of naïve (albeit not memory) T-cells (11), coupled to the ability of repetitive antigen-specific stimulation of the TCR to elicit $\operatorname{Tr} 1$-like phenotypic features $(12,13)$ likely play a significant role in this outcome. Nevertheless, in vitro experiments have suggested that repetitive engagement of cognate TCRs by pMHCII-NPs, albeit necessary, is not sufficient to fully induce the differentiation of autoantigen-experienced cells into $\operatorname{Tr} 1$ cell progeny. It remains unclear if pMHCII-NPs can induce Tr1 cell formation from any cognate autoantigen-experienced CD4+ Tcell subset regardless of cell differentiation status, or only from a specific Tr1-poised precursor cell type. Ongoing transcriptional and epigenetic studies will shed light into the mechanistic underpinnings of this differentiation process.

\section{KEY pMHC-NP ENGINEERING DESIGN PRINCIPLES}

The above observations prompted us to define what were the key pMHC-NP engineering design variables, to guide the development of next generation nanomedicines suitable for drug development and clinical translation. Extensive experimentation with various inorganic NP types (largely iron oxide-based) demonstrated that the biological activity of pMHC-NPs produced with NPs of a given size is a function of pMHC valency (number of pMHC monomers per NP) (14). In vitro studies using NPs of different sizes further indicated that the "optimal" pMHC valency values increased with $\mathrm{NP}$ size, indicating that $\mathrm{pMHC}$ density (number of pMHCs/ surface area), rather than pMHC valency (absolute number of $\mathrm{pMHCs} / \mathrm{NP}$, regardless of NP size), is the most critical parameter (14). That is, it is not the absolute number of pMHC monomers per NP that determines potency, but rather the density of these 
pMHCs on the NP surface, such that NPs of different sizes carrying identical numbers of pMHCs will have different potencies. When tested on reporter Jurkat cells expressing cognate TCRs, these compounds lacked significant TCR triggering activity below a certain $\mathrm{pMHC}$ valency/density threshold. The TCR signaling potency of these compounds increased exponentially in response to relatively small increases in $\mathrm{pMHC}$ valency/density, starting at the $\mathrm{pMHC}$ valency/density threshold and ending at a "minimal optimal" pMHC valency/ density value, at which the TCR signaling intensity plateaued. Substantial increases in pMHC valency/density above this minimal optimal valency did not result in significantly higher potency (14). These observations suggested that NPs displaying threshold and supra-threshold pMHC densities somehow promote cooperative TCR signaling.

In vitro, compounds displaying threshold and suprathreshold pMHC valencies/densities elicited very rapid (within $2 \mathrm{~h}$ ), vigorous and sustained ( $>24 \mathrm{~h}$ ) TCR signals, as compared to optimal concentrations of an agonistic CD $3 \epsilon$ mAb or PMA/ ionomycin, which triggered much slower responses that peaked at $14 \mathrm{~h}$ and progressively decreased afterwards. Furthermore, imaging of pMHC-NP/T-cell interactions via transmission electron microscopy, super-resolution microscopy and scanning electron microscopy revealed that pMHC-NPs bind cognate T-cells as clusters of several NPs spanning $\sim 100-150 \mathrm{~nm}$ that progressively grew to $\sim 400 \mathrm{~nm}$, culminating in internalization of the NPs in intracellular vesicles, starting $\sim 3 \mathrm{~h}$ after binding. Importantly, cluster formation was only observed when using NPs coated at threshold and supra-threshold pMHC valencies/densities. Thus, pMHC-NPs function as sustained TCR nanocluster-binding and microcluster-triggering devices (Figure 1A). Collectively, these observations indicated that small NPs coated at the highest possible pMHC densities, allowing a nearperfect alignment of pMHCs on the NP and cognate TCRs on target T-cells, represent the most optimal design (14) (Figure 1A).

Subsequent in vivo experimentation with pMHCII-NPs suggested that whereas pMHC density regulates the efficiency of $\operatorname{Tr} 1$ cell formation, pMHC dose controls the magnitude of $\operatorname{Tr} 1$ cell expansion, indicating that $\mathrm{pMHC}$ density and $\mathrm{pMHC}$ dose have separate roles (14).

In terms of translation, the chemistry employed in the manufacture of iron oxide-based NPs is scalable. It is worth noting that, when used as MRI contrast agents in humans, these NP compounds are immunologically inert, biocompatible and safe. With regards to their pMHC-coated iron oxide NP counterparts, we have shown that such compounds have no off-target toxicity in zebrafish embryos, and do not cause hematological, biochemical or histological abnormalities in mice (14).

Our first generation pMHC compounds involved the expression of recombinant pMHC molecules in E. coli or Drosophila S2 cells followed by purification using 6xHis and/or streptag affinity chromatography. Low yields and the need to incorporate artificial affinity purification tags into the pMHC design represented significant obstacles for clinical translation.
Expression in Chinese Hamster Ovary (CHO) cells and reengineering of pMHC heterodimers as knob-into-hole-based Fc fusions addressed these limitations; KIH-based pMHC molecules are expressed at much higher yields than pMHCIIs heterodimerized using leucine zippers and can be purified to the desired levels of purity using protein A chromatography and additional polishing steps routinely used in the purification of biologics (15).

\section{BYSTANDER IMMUNOREGULATION MEDIATED BY REGULATORY CELL NETWORKS ARISING DOWNSTREAM OF TR1 CELL FORMATION}

Studies in T1D (and later confirmed in other disease models) showed that pMHCII-NP-induced/expanded Tr1 cells suppressed the pro-inflammatory and antigen presentation capacities of local and proximal (i.e. in pancreas-draining lymph nodes) autoantigen-loaded dendritic cells (DCs) and myeloid APCs in an Interleukin-10 (IL-10)- and Tumor Growth Factor beta (TGF $\beta$ )-dependent manner. Furthermore, recruitment of these antigen-specific $\operatorname{Tr} 1$ cells into the pancreasdraining lymph nodes of the treated mice promoted the formation/recruitment of interleukin-10 (IL-10)-producing CD1d $\mathrm{d}^{\text {high }} / \mathrm{CD} 5+$ B-cells (Figure 1B). Transfer of cognate peptide-pulsed B-cells from donors expressing an IL-10 reporter transgene into treated recipients elicited de novo IL-10 expression in the donor B-cells, indicating that Breg cell formation in the pancreatic lymph nodes of these mice was induced by cognate Tr1-B-cell interactions. Antibody-mediated cytokine blockade demonstrated that, unlike APC suppression, Tr1-driven Breg cell formation was IL-21-dependent but IL-10 and TGF $\beta$-independent. Both, BDC2.5mi//A ${ }^{\mathrm{g} 7}$ tetramer+ T-cells and pancreatic lymph node-derived B-cells from treated donors could blunt the transfer of T1D to NOD.scid mice by splenocytes from untreated NOD mice, demonstrating the independent immunoregulatory activity of both cell types. Simultaneous transfer of both cell types had maximal (synergistic) therapeutic activity. Thus, pMHCII-NP therapy elicits the formation of disease-specific regulatory cell networks capable of restoring immune homeostasis.

\section{PMHCII-NPs DISPLAYING EPITOPES FROM LIVER AUTOIMMUNE DISEASE- RELEVANT UBIQUITOUS AUTOANTIGENS}

Autoimmunity in the liver manifests itself through various diseases, including primary biliary cholangitis ( $\mathrm{PBC}$ ), primary sclerosing cholangitis (PSC) and autoimmune hepatitis (AIH). In these diseases, unlike those discussed above, the autoimmune response recognizes ubiquitously expressed autoantigens, such as the mitochondrial pyruvate dehydrogenase complex-E2 component 
A

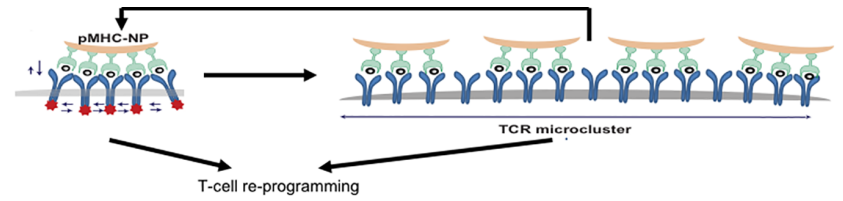

B Tissue-specific autoantigen-based pMHCII-NP

C Ubiquitous autoantigen-based pMHCII-NP

Autoimmune
inflammation,

Autoimmune
inflammation, inflammation,
cell destruction

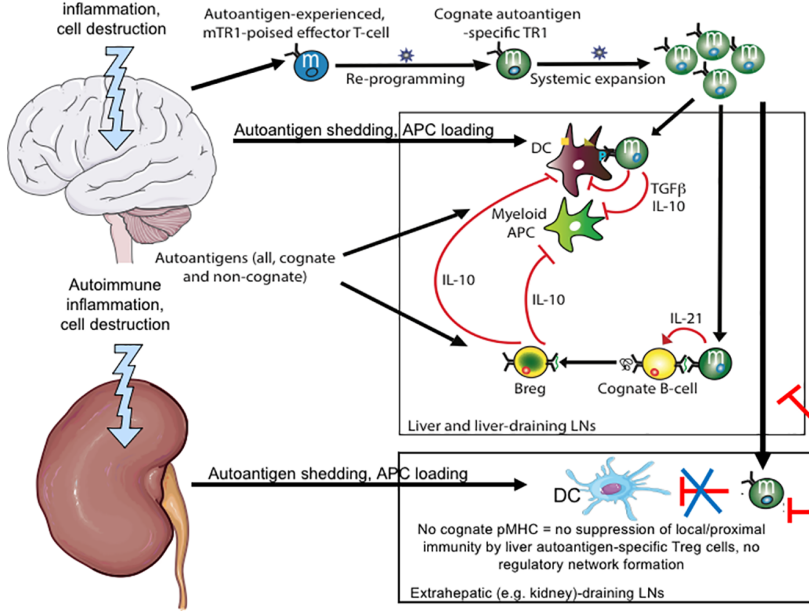
inflammation,
cell destruction

5

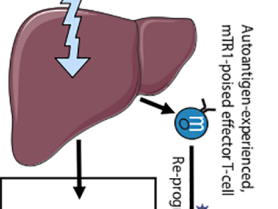
shedding (e.g.
PDC-E2, ubiquitous), APC

* loading

FIGURE 1 | pMHCII-NPS: engineering and mechanisms. (A) pMHC-based nanomedicines function as antigen-receptor microclustering devices. Left, NP coated with a high-density array of mono-specific pMHC monomers elicits the simultaneous activation of multiple contiguous T-cell receptors, resulting in powerful signal amplification, cytoskeletal rearrangements, and TCR cluster formation. In turn, this increases the avidity of the target T-cell for additional incoming pMHCNPs, further amplifying TCR signaling. Collectively, this profound, sustained and repetitive pMHC-NP engagement triggers T-cell re-programming through as yet unclear mechanisms. (B) pMHCll-based nanomedicines displaying epitopes from tissue-specific autoantigens [e.g. the central nervous system (CNS) in this cartoon] trigger the formation and subsequent expansion of CNS-specific T-regulatory type 1 (Tr1)-like cells. These cells biodistributed systemically, but exclusively undergo productive activation upon recognition of cognate pMHCII on professional APCs capable of delivering co-stimulatory signals (i.e. autoantigen-loaded DCs in the CNS or the CNS-draining lymph nodes). This elicits the local production of regulatory cytokines capable of suppressing autoantigen presentation to other autoreactive T-cell specificities. In addition, these cytokines recruit and locally re-program other immune cell types (e.g. B-cells) into cells with regulatory properties (Breg cells in this case). Collectively, these regulatory cell networks suppress local inflammatory processes, blunt disease progression and promote tissue repair. When these pMHCII-NP-induced CNS-specific Tr1 cells encounter APCs in other tissues/organs (e.g. kidney) lacking the Tr1 cells' cognate autoantigen, they fail to engage the APC, hence to undergo productive activation. (C) pMHCII-based nanomedicines displaying epitopes from ubiquitous autoantigens (e.g. liver autoimmune disease-relevant) trigger the formation and subsequent expansion of Tr1-like cells that have the potential to suppress autoimmune responses in more than one tissue/organ.

(PDC-E2) in PBC; or nuclear, cytoplasmic, or Golgi-enriched proteins, such as F-actin, formimidoyltransferase cyclodeaminase (FTCD), or cytochrome P450 (CYPD2D6) in AIH; or tropomyosin isoform 5 (hTM5) in PSC, among others. In addition, there is a significant subgroup of patients in which liver autoimmunity has features of both, cholestasis and autoimmune hepatitis, suggesting that autoimmune responses against certain autoantigenic targets in a given liver autoimmune disease (e.g. cholangitis) can spread to anatomic liver structures that are preferentially targeted in other liver autoimmune diseases (e.g. hepatitis). These observations begged the question of whether autoimmune liver disease-relevant pMHCII-NP compounds would be disease-specific (e.g. against $\mathrm{PBC}$ ) or pan-liver autoimmune disease-specific (e.g. capable of blunting different liver autoimmune diseases).

Systemic delivery of two different PBC-relevant compounds (PDC-E2 ${ }_{166-181} / \mathrm{IA}^{\mathrm{g} 7}$ - and PDC-E2 ${ }_{82-96} / \mathrm{IA}^{\mathrm{g} 7}-\mathrm{NPs}$ ) blunted the progression of liver autoimmunity in NOD.c3c4 mice and (NODxB6.Ifng ARE-Del ${ }^{-1-}$ ) F1 mice, which spontaneously develop a form of liver autoimmunity that closely resembles human PBC (4) (Table 1). These compounds also blunted the progression of spontaneous PSC in $A b c b 4$ knockout mice and the progression of experimental AIH in NOD mice (induced by infection with an adenovirus encoding the human $\mathrm{AIH}$-relevant autoantigen FTCD) (4) (Table 1). Likewise, both $\mathrm{CYPD}_{398-412} / \mathrm{IA}^{\mathrm{g} 7}-\mathrm{NPs}$ and PDC-E2 ${ }_{166-181} / \mathrm{IA}^{\mathrm{g} 7}$-NPs (AIH and PBC-relevant nanomedicines, respectively) blunted Ad-hFTCD-induced AIH in NOD mice as efficiently as mFTCD $58-72 / \mathrm{IA}^{\mathrm{g} 7}$-NPs. $\mathrm{CYPD}_{398-412} / \mathrm{IA}^{\mathrm{g} 7}-\mathrm{NPs}$ could also blunt the progression of PSC in NOD.Abcb4 $4^{-1-}$ mice (4) (Table 1). In these models, the various pMHCII-NPs suppressed disease by eliciting the formation and expansion of cognate Tr1-like CD4+ T-cells, the suppression of pro-inflammatory and antigenpresenting capacities of local and proximal APCs, and the formation/ recruitment of Breg cells (4) (Figure 1C). Importantly, therapy with these compounds suppressed liver autoimmunity without impairing immunity against Influenza, Vaccinia, or L. monocytogenes infections or against allogeneic metastatic liver tumors (4). 
Collectively, the above observations support the view that the tissue damage arising in response to a liver autoimmune disease initiated by autoreactive $\mathrm{T}$-cells recognizing specific diseaserelevant autoantigen(s) (e.g. PDC-E2 in $\mathrm{PBC}$ ) results in the priming and recruitment of $\mathrm{T}$-cell specificities targeting other autoantigens. Our work indicates that these secondary T-cell specificities can also be harnessed by pMHCII-NPs to blunt disease progression, as we had previously documented in EAE (Table 1). Thus, pMHCII-NPs need not have to target diseaseinitiating or prevalent autoreactive T-cell specificities to elicit therapeutic activity in a given autoimmune disease.

\section{TREATMENT OF EXTRA-HEPATIC AUTOIMMUNITY BY PMHCII-NPS DISPLAYING UBIQUITOUS AUTOANTIGENIC EPITOPES}

The above observations suggested that pMHCII-NPs displaying ubiquitously expressed epitopes might also have therapeutic activity against extra-hepatic autoimmune diseases. For these compounds to work, the epitopes derived from the ubiquitous protein would at least have to participate in the autoimmune response without necessarily playing a significant role in tissue destruction. Furthermore, the corresponding antigenic epitopes would have to be presented by professional APCs in amounts sufficient to trigger the activation of cognate CD4+ T-cells, to render them capable of responding to cognate pMHCIINP treatment.

We sought to first investigate these assumptions by treating NOD mice with PDC-E2 $166-181 / \mathrm{IA}^{\mathrm{g} 7}-\mathrm{NPs}$ (PBC-relevant) and $\mathrm{CYPD}_{398-412} / \mathrm{IA}^{\mathrm{g} 7}$-NPs (AIH-relevant). Neither of these two compounds triggered the expansion of cognate Tr1-like CD4+ Tcells, suggesting that pancreatic beta cells either did not shed the corresponding antigenic epitopes, or did so in amounts insufficient to generate epitope-experienced CD4+ T-cells (Table 1). Diphtheria toxin (DT)-induced killing of $\sim 50 \%$ beta cells of NOD mice expressing an X-chromosome-linked rat-insulin promoter-driven human diphtheria toxin receptor (hDTR) transgene rendered these mice responsive to $\mathrm{PDC}-\mathrm{E} 2_{166-181} / \mathrm{IA}^{\mathrm{g} 7}-\mathrm{NPs}, \mathrm{CYPD}_{398-412} / \mathrm{IA}^{\mathrm{g} 7}$ NPs (PBC/AIH-relevant) and BDC2.5/IA ${ }^{\mathrm{g} 7}$-NPs (T1D-specific), but not $\mathrm{MOG}_{36-50} / \mathrm{IA}^{\mathrm{g} 7}$-NPs (EAE-specific, not expressed in pancreatic beta cells) (Table 1). The cognate pMHCII-NPinduced Tr1 cells that accumulated in the liver and pancreasdraining lymph nodes of these mice suppressed the activation of non-cognate beta-cell-autoreactive T-cells by local APCs, thus demonstrating that (1) pMHCII-NP-induced Tr1 cell formation requires autoantigen-experienced T-cells (2) that NOD mice harbor T-cells targeting ubiquitously-expressed antigens, and (3) that the priming of such cells requires antigen shedding (5) (Figures 1B, C).

We then compared the ability of PDC-E2 ${ }_{166-181} / \mathrm{IA}^{\mathrm{g} 7}-\mathrm{NPs}$ and $\mathrm{CYPD}_{398-412} / \mathrm{IA}^{\mathrm{g} 7}-\mathrm{NPs}$ (vs. BDC2.5/IA ${ }^{\mathrm{g} 7}-\mathrm{NPs}$ and $\mathrm{MOG}_{36-50} /$ $\mathrm{IA}^{\mathrm{g} 7}$-NPs as negative and positive controls, respectively), or PDCE2 ${ }_{94-108} / \mathrm{IA}^{\mathrm{b}}$-NPs and $\mathrm{CYPD}_{353-367} / \mathrm{IA}^{\mathrm{b}}$-NPs to blunt $\mathrm{MOG}_{36-55^{-}}$ induced $\mathrm{EAE}$ in NOD and $\mathrm{C} 57 \mathrm{BL} / 6$ mice, respectively. These experiments indicated that, upon oligodendrocyte damage, both
PDC-E2 and CYPD2D6 (but not BDC2.5, which is not expressed in the CNS) are delivered to proximal APCs for autoreactive CD4+ T-cell priming, enabling Tr1 cell generation by cognate pMHCIINPs, their recruitment to the CLNs, and suppression of EAE (Table 1).

Subsequent experiments using $\mathrm{MOG}_{38-49} / \mathrm{IA}^{\mathrm{b}}$-, PDC-E2 ${ }_{94-108} /$ $\mathrm{IA}^{\mathrm{b}}$-, $\mathrm{CYPD}_{353-367} / \mathrm{IA}^{\mathrm{b}}$ - and $\mathrm{Fla}_{462-472} / \mathrm{IA}^{\mathrm{b}}$-NPs (as a negative control) in B6 mice having Ad-hFTCD-induced AIH and/or EAE revealed that $\operatorname{Tr} 1$ cell recruitment and therapeutic effects require local autoantigen expression (Figure 1B). Interestingly, liver inflammation in mice simultaneously having both EAE and $\mathrm{AIH}$ sequestered the ubiquitous antigen-specific Tr1 cells away from the CNS, abrogating their ability to blunt CNS autoimmunity (Table 1). Resolution of liver inflammation released these cells for recruitment to the CLNs, enabling them to blunt EAE (5) (Table 1) (Figure 1C). When we superimposed EAE onto the more aggressive, chronic form of liver autoimmunity that develops in NOD.c3c4 mice, the three pMHCII-NPs tested (PDC-E2 $166-181$ / $\mathrm{IA}^{\mathrm{g} 7}-, \mathrm{CYPD}_{398-412} / \mathrm{IA}^{\mathrm{g} 7}$ - and $\left.\mathrm{MOG}_{36-50} / \mathrm{IA}^{\mathrm{g} 7}-\mathrm{NPs}\right)$ had pharmacodynamic activity but lacked therapeutic activity; liver inflammation in these mice retained antigen-specific $\operatorname{Tr} 1$ cells non-specifically (5) (Table 1).

To better understand how pMHCII-NP-expanded Tr1 cells traffic to multiple sites of inflammation (in co-morbid mice), we developed a mathematical model composed of a system of nonlinear ordinary differential equations (16). We compartmentalized the model into separate cell pools, each representing the organs under consideration, and evaluated the validity of the above experimental observations to understand the interplay between Tr1-cell allocation and pMHCII-NP therapeutic efficacy. In agreement with the experimental data, this model suggested that cognate autoantigen expression and local Tr1-cell retention are key determinants of effective regulatory-cell function downstream of pMHCII-NP therapy. Tissues competing for the same $\operatorname{Tr} 1$ resource (i.e. in comorbid mice) may give rise to competitive autoimmunity where neither tissue will recruit a sufficient number of Tr1 cells beyond the suppression threshold (due to either impaired recruitment/retention or inefficient $\operatorname{Tr} 1$ suppressive potential) (16).

Collectively, these data indicated that (1) autoreactive T-cells targeting ubiquitous antigens can be awakened by antigen shedding from different cells/tissues (Figure 1C); (2) local autoantigen expression is required for the regulatory activity of antigen-specific Tr1-like cells (Figures 1B, C): (3) liver inflammation has the potential to non-specifically draw $\mathrm{T}$ regulatory cells away from sites of cognate autoantigen expression and autoimmune inflammation.

\section{PHARMACODYNAMIC ACTIVITY OF HUMAN AUTOIMMUNE DISEASE- RELEVANT PMHCII-NPS IN HUMANIZED MICE}

The pharmacodynamic activity of murine pMHCII-NPs in mice could be replicated in NOD.scid/Il2 $\mathrm{rg}^{-/-}$(NSG) mice engrafted with peripheral blood mononuclear cells (PBMCs) from patients. 
Treatment of NSG mice humanized with PBMCs from DRB $1^{\star} 0301+$ and/or DRB $1^{\star} 0401+$ T1D patients with NPs displaying human $\mathrm{IGRP}_{13-25}-\mathrm{DRB} 1^{\star} 0301$ or human preproinsulin $(\mathrm{PPI})_{76-90} / \mathrm{DRB} 1^{\star} 0401$ complexes resulted in the expansion of cognate IL-10-producing CD4+ T-cells coexpressing the $\operatorname{Tr} 1$ cell markers CD49b and LAG-3 (Table 1). Similar observations were made in NSG hosts reconstituted with PBMCs from $\mathrm{DRB} 4{ }^{\star} 0101+$ or $\mathrm{DRB} 1^{\star} 0801+\mathrm{PBC}$ patients in response to treatment with $\mathrm{PDC}-\mathrm{E} 2{ }_{122-135} / \mathrm{DRB} 4^{\star} 0101-$, $\mathrm{PDC}$ $\mathrm{E} 2_{249-262} / \mathrm{DRB} 4^{\star} 0101-$, and $\mathrm{PDC}-\mathrm{E} 2{ }_{629-643} / \mathrm{DRB} 1^{\star} 0801-\mathrm{NPs}$ (Table 1). These observations support the translational potential of these compounds for the treatment of human autoimmunity, and introduce a preclinical validation tool for human pMHCII-NP candidates. Further refinement of this model, including the use of mice with a more developed humanized peripheral immune system, will further facilitate the pre-clinical evaluation of clinical candidates.

\section{CONCLUDING REMARKS}

The therapeutic activity of NPs coated with disease-relevant pMHC molecules was an accident of curiosity-driven research that suggested that these compounds, initially designed for T-cell deletional therapy, could elicit bystander immunoregulation. Studies on a significant number of spontaneous and experimental autoimmune disease models using numerous pMHC-NP compounds have established the therapeutic potential of this approach to treat a whole host of autoimmune disorders in a disease-specific manner without compromising normal immunity. We have defined the key NP and $\mathrm{PMHC}$ engineering principles that are responsible for pharmacodynamic activity and have dissected mechanisms underlying therapeutic activity, namely $\operatorname{Tr} 1$ cell formation from an antigen-experienced precursor type, followed by systemic expansion, recruitment to the target tissue and formation of regulatory cell networks responsible for sustained and comprehensive therapeutic activity. The composition of these regulatory cell networks and the molecular cues responsible for their assembly and homeostasis likely vary as a function of disease type and organ. In liver autoimmunity, for example, the antigen-specific Trl cells and Breg cells that arise in response to pMHC-NP therapy cooperatively induce the recruitment and re-programming of neutrophils into a regulatory cell subset that resembles granulocyte myeloid-derived suppressor cells (MDSCs). The nature of the autoantigen-experienced T-cell type that gives rise to cognate $\operatorname{Tr} 1$ cells in response to pMHCII-NP therapy remains unclear, and so do the mechanisms via which sustained TCR signaling re-programs this precursor cell type into a Tr1 cell type.

The ability of these compounds to suppress autoantigen-loading APCs may explain why they spare normal immune responses to pathogens. The short half-life of dendritic cells in vivo implies that de novo suppression of newly recruited (non-autoantigen-loaded) APCs is required for sustained immunoregulation. On the other hand, this allows new, non-immunosuppressed APCs to process and present pathogen-derived antigens to pathogen-specific T-cell specificities. In addition, during a local infection, antigens derived from local pathogens likely overwhelm the APCs' antigen presentation machinery, diluting expression of the Trl's cognate pMHC below the threshold required for Tr1 cell-induced APC immunoregulation. Upon clearance of the infection, new incoming APCs would then regain the ability to present the Trl's cognate pMHC, allowing these $\operatorname{Tr} 1$ cells to resume their antiinflammatory activity.

At the translational level, we have made significant progress in candidate pMHCII identification and selection for specific autoimmune diseases. Our experimental work in mice has suggested that most, if not all, CD4+ T-cell specificities recognizing autoantigenic epitopes expressed by the target tissue of a given autoimmune disease can be re-programmed into Tr1 cells in vivo via pMHCII-NP therapy. Ideal clinical candidates are those displaying epitopes from prevalent tissue-specific autoantigens in the context of allelic MHCII types expressed by a significant fraction of the patient population. For autoimmune diseases with strong HLA class II associations, such as T1D or Celiac Disease, the choice of HLA type is straightforward. For diseases in which there is not a strong HLA class II allelic bias, the use of MHCII molecules encoded in oligomorphic HLA class II loci, such as DRB3, DRB4 and DRB5 loci is desirable. Up to three different pMHC-NP compounds would be sufficient to treat $>80 \%$ of the patient population for any given autoimmune disorder. Notwithstanding the progress to date, candidate pMHCII selection remains a bottleneck that would benefit from the availability of improved, higher throughput methods capable of enumerating the frequency of defined pHLAII specificities (as opposed to peptide specificities regardless of HLA restriction) in patients' peripheral blood samples. Although we have provided compelling evidence supporting translational potential, we do not yet know whether these compounds will be effective in clinical trials.

Lastly, the work done to date begs the question of whether T-cell types other than CD8+ and CD4+ T-cells, such as invariant Natural Killer T-cells or Mucosal Associated Invariant T-cells, can also be re-programmed into autoimmune disease-suppressing cell types using MHC-based nanomedicines. The next few years should provide answers to these outstanding questions.

\section{AUTHOR CONTRIBUTIONS}

The manuscript was written and edited by both co-authors. All authors contributed to the article and approved the submitted version.

\section{FUNDING}

The authors' work summarized here was funded by the Canadian Institutes of Health Research (CIHR), Diabetes Canada, the Crohn's and Colitis Foundation of Canada, the Multiple Sclerosis Society of Canada (MSSC), ISCIII and FEDER (PIE14/00027, PI15/0797), REEM (Red Española de Esclerosis Múltiple), NEURON-ERANET (European Research Projects on Neuroinflammation; NEURON7-FP-715-018), the Praespero Foundation, the Ministerio de Economia y Competitividad of 
Spain (MINECO; RD16/0015/0020), and Generalitat de Catalunya (SGR and CERCA Programmes). The JMDRC is supported by Diabetes Canada.

\section{REFERENCES}

1. Serra P, Santamaria P. Antigen-specific therapeutic approaches for autoimmunity. Nat Biotechnol (2019) 37:238-51. doi: 10.1038/s41587-0190015-4

2. Tsai S, Shameli A, Yamanouchi J, Clemente-Casares X, Wang J, Serra P, et al. Reversal of autoimmunity by boosting memory-like autoregulatory $\mathrm{T}$ cells. Immunity (2010) 32:568-80. doi: 10.1016/j.immuni.2010.03.015

3. Clemente-Casares X, Blanco J, Ambalavalan P, Yamanouchi J, Singha S, Fandos C, et al. Expanding antigen-specific regulatory networks to treat autoimmunity. Nature (2016) 530:434-40. doi: 10.1038/nature16962

4. Umeshappa CS, Singha S, Blanco J, Shao K, Nanjundappa RH, Yamanouchi J, et al. Suppression of a broad spectrum of liver autoimmune pathologies by single peptide-MHC-based nanomedicines. Nat Commun (2019) 10:2150. doi: 10.1038/s41467-019-09893-5

5. Umeshappa CS, Mbongue J, Singha S, Mohapatra S, Yamanouchi J, Lee JA, et al. Ubiquitous antigen-specific $\mathrm{T}$ regulatory type 1 cells variably suppress hepatic and extrahepatic autoimmunity. J Clin Invest (2020) 130:1823-9. doi: 10.1172/JCI130670

6. Amrani A, Verdaguer J, Serra P, Tafuro S, Tan R, Santamaria P. Progression of autoimmune diabetes driven by avidity maturation of a T-cell population. Nature (2000) 406:739-42. doi: 10.1038/35021081

7. Han B, Serra P, Amrani A, Yamanouchi J, Maree A, Edelstein-Keshet L, et al. Prevention of diabetes by manipulation of anti-IGRP autoimmunity: high efficiency of a low-affinity peptide. Nat Med (2005) 11:645-52. doi: 10.1038/nm1250

8. Han B, Serra P, Yamanouchi J, Amrani A, Elliott J, Dickie P, et al. Developmental control of CD8+ $\mathrm{T}$ cell-avidity maturation in autoimmune diabetes. J Clin Invest (2005) 115:1879-87. doi: 10.1172/JCI24219

9. Burton BR, Britton GJ, Fang H, Verhagen J, Smithers B, Sabatos-Peyton CA, et al. Sequential transcriptional changes dictate safe and effective antigen-specific immunotherapy. Nat Commun (2014) 5:4741. doi: 10.1038/ncomms5741

10. Gagliani N, Magnani CF, Huber S, Gianolini ME, Pala M, Licona-Limon P, et al. Coexpression of CD49b and LAG-3 identifies human and mouse T regulatory type 1 cells. Nat Med (2013) 19:739-46. doi: 10.1038/nm.3179

\section{ACKNOWLEDGMENTS}

We thank the members of our laboratories for their contributions.

11. Boesteanu AC, Katsikis PD. Memory T cells need CD28 costimulation to remember. Semin Immunol (2009) 21:69-77. doi: 10.1016/j.smim.2009.02.005

12. Gabrysova L, Nicolson KS, Streeter HB, Verhagen J, Sabatos-Peyton CA, Morgan DJ, et al. Negative feedback control of the autoimmune response through antigen-induced differentiation of IL-10-secreting Th1 cells. J Exp Med (2009) 206:1755-67. doi: 10.1084/jem.20082118

13. Gabrysova L, Wraith DC. Antigenic strength controls the generation of antigen-specific IL-10-secreting T regulatory cells. Eur J Immunol (2010) 40:1386-95. doi: 10.1002/eji.200940151

14. Singha S, Shao K, Yang Y, Clemente-Casares X, Sole P, Clemente A, et al. Peptide-MHC-based nanomedicines for autoimmunity function as T-cell receptor microclustering devices. Nat Nanotechnol (2017) 12:701-10. doi: 10.1038/nnano.2017.56

15. Serra P, Garabatos N, Singha S, Fandos C, Garnica J, Sole P, et al. Increased yields and biological potency of knob-into-hole-based soluble MHC class II molecules. Nat Commun (2019) 10:4917. doi: 10.1038/s41467019-12902-2

16. Jamaleddine H, Santamaria P, Khadra A. Quantifying immunoregulation by autoantigen-specific T-regulatory type 1 cells in mice with simultaneous hepatic and extra-hepatic autoimmune disorders. Immunology (2020) 161:209-29. doi: 10.1111/imm.13241

Conflict of Interest: PS is the scientific founder of Parvus Therapeutics Inc. and has a financial interest in the company.

The remaining author declares that the research was conducted in the absence of any commercial or financial relationships that could be construed as a potential conflict of interest.

Copyright (c) 2021 Serra and Santamaria. This is an open-access article distributed under the terms of the Creative Commons Attribution License (CC BY). The use, distribution or reproduction in other forums is permitted, provided the original author(s) and the copyright owner(s) are credited and that the original publication in this journal is cited, in accordance with accepted academic practice. No use, distribution or reproduction is permitted which does not comply with these terms. 